\title{
CTA Protocols in a Telestroke Network Improve Efficiency for Both Spoke and Hub Hospitals
}

(D) A.T. Yu, (D).W. Regenhardt, (D) C. Whitney, (D) L.H. Schwamm, (D)A.B. Patel, (D) C.J. Stapleton, (D) A. Viswanathan, (D).A. Hirsch, (D) M. Lev, and (D)T.M. Leslie-Mazwi

\begin{abstract}
BACKGROUND AND PURPOSE: Telestroke networks support screening for patients with emergent large-vessel occlusions who are eligible for endovascular thrombectomy. Ideal triage processes within telestroke networks remain uncertain. We characterize the impact of implementing a routine spoke hospital CTA protocol in our integrated telestroke network on transfer and thrombectomy patterns.
\end{abstract}

\begin{abstract}
MATERIALS AND METHODS: A protocol-driven CTA process was introduced at 22 spoke hospitals in November 2017. We retrospectively identified prospectively collected patients who presented to a spoke hospital with National Institutes of Health Stroke Scale scores $\geq 6$ between March 1, 2016 and March 1, 2017 (pre-CTA), and March 1, 2018 and March 1, 2019 (post-CTA). We describe the demographics, CTA utilization, spoke hospital retention rates, emergent large-vessel occlusion identification, and rates of endovascular thrombectomy.

RESULTS: There were 167 patients pre-CTA and 207 post-CTA. The rate of CTA at spoke hospitals increased from 15\% to $70 \%$ $(P<.001)$. Despite increased endovascular thrombectomy screening in the extended window, the overall rates of transfer out of spoke hospitals remained similar (56\% versus $54 \% ; P=.83$ ). There was a nonsignificant increase in transfers to our hub hospital for endovascular thrombectomy $(26 \%$ versus $35 \% ; P=.12$ ), but patients transferred $>4.5$ hours from last known well increased nearly 5 fold $(7 \%$ versus $34 \% ; P<.001)$. The rate of endovascular thrombectomy performed on patients transferred for possible endovascular thrombectomy more than doubled (22\% versus $47 \% ; P=.011)$.

CONCLUSIONS: Implementation of CTA at spoke hospitals in our telestroke network was feasible and improved the efficiency of stroke triage. Rates of patients retained at spoke hospitals remained stable despite higher numbers of patients screened. Emergent large-vessel occlusion confirmation at the spoke hospital lead to a more than 2-fold increase in thrombectomy rates among transferred patients at the hub.
\end{abstract}

ABBREVIATIONS: EVT = endovascular thrombectomy; LKW = last known well; SH = spoke hospital; ELVO = emergent large-vessel occlusion; LVO = large-vessel occlusion; NIHSS = National Institutes of Heath Stroke Scale

$\mathbf{T}$ elestroke supports thrombolytic use and screening for patients with emergent large-vessel occlusion (ELVO) across stroke systems of care. ${ }^{1-3}$ ELVOs represent a minority of acute stroke presentations but produce most morbidity and

Received June 23, 2020; accepted after revision October 3.

From the Departments of Neurology (A.T.Y., R.W.R., C.W., L.H.S., A.V., T.M.L.-M.) Neurosurgery (R.W.R., A.B.P., C.J.S., T.M.L.-M.), and Radiology (J.A.H., M.L.), Massachusetts General Hospital, Boston, Massachusetts.

R.W.R. is supported by NIH-NINDS R25NS065743

Paper previously presented at: International Stroke Conference 2020, February 19; Los Angeles, California.

Please address correspondence to Andrew Yu, MD, Wang Ambulatory Care Center, 8th Floor, Suite 835, 15 Parkman St, Boston, MA 02214; e-mail: ayul2@bwh.harvard.edu; @andrewyu8; @rwregen; @braindoc_mgh; @amanbpatel; @joshuaahirsch; @michaellevmd

- Indicates open access to non-subscribers at www.ajnr.org

Indicates article with online supplemental data.

http://dx.doi.org/10.3174/ajnr.A6950 mortality in ischemic stroke and are therefore a critical area of focus within stroke care systems. ${ }^{4-6}$ For patients with ELVO, the current target of acute stroke therapy is penumbral salvage, which relies on numerous factors, the most important of which is timely reperfusion. ${ }^{7-9}$ The powerful therapeutic effect of early reperfusion ( $<6$ hours from last known well [LKW]) through endovascular thrombectomy (EVT) for patients with ELVO is now well established. ${ }^{10-12}$ The time window for treatment expanded with the demonstration of benefit of reperfusion in patients between 6 and 24 hours from LKW, a demographic for which previously no therapeutic intervention was available. ${ }^{13-14}$ Selection for thrombectomy in both early and late treatment windows required identification of ELVO by noninvasive imaging in all recent trials.

The expansion of treatment with EVT up to 24 hours from LKW has dramatically increased the pool of patients to screen. Current telestroke care system approaches require the emergent 
transfer of patients from spoke hospitals (SHs), which lack the ability to perform thrombectomy, to hub hospitals, which are thrombectomy capable. Ideal triage processes within telestroke networks remain uncertain and may vary based on geographic region. Here we describe the effects of the implementation of a routine $\mathrm{SH}$ CTA protocol within our integrated telestroke network on $\mathrm{SH}$ retention rates and hub hospital thrombectomy rates.

\section{MATERIALS AND METHODS}

We introduced a protocol-driven CTA process at $22 \mathrm{SHs}$ within our telestroke network in the northeastern United States in November 2017. The CTA protocol specified CTA indications, acquisition parameters, and methods for image reconstruction (Supplemental Online Data) to provide a standardized imaging approach across the telestroke network. Before this CTA protocol, in the pre-CTA era, transfer for EVT consideration occurred mainly driven by stroke severity (NIHSS score $\geq 6$ points), time from LKW ( $\leq 6$ hours), ASPECTS score $\geq 6$ on noncontrast CT, and mRS score $0-2$, based primarily on the American Heart Association guidelines at that time. ${ }^{15}$ After implementation of the protocol, the post-CTA era, all patients presenting to an $\mathrm{SH}$ with NIHSS score $\geq 6$ points and $<24$ hours from LKW were required to undergo CTA imaging in addition to and immediately after their noncontrast head CT. Head and neck CTA with reconstruction of thick, overlapping axial MIPs was a requirement in the protocol. Additional imaging suggested by the protocol but at the discretion of the site included CTA delays of the head and neck and sagittal and/or coronal MIPs. After acquisition, this imaging was shared through a cloud-based platform between the $\mathrm{SH}$ and the hub. Additional criteria for transfer for EVT consideration in the post-CTA era were based on updated guidelines and included ASPECTS score $\geq 6$ and mRS score $0-2 .{ }^{16}$

We retrospectively identified patients who presented to an SH with NIHSS scores $\geq 6$ points between March 1, 2016 and March 1, 2017 (pre-CTA), and between March 1, 2018 and March 1, 2019 (post-CTA). A 1-year gap between our pre-CTA and post-CTA cohorts was used to allow enough time for uniform adoption of the CTA protocol across all SHs. Patients were identified through our telestroke consult log, which records consecutive telestroke consults placed within our network. Within each cohort, we analyzed baseline demographics, risk factors, CTA utilization, ELVO identification, and transfer to a hub hospital. We defined off-hour telestroke utilization as a consult start time between 7 PM and 7 AM regardless of the day of the week. We obtained patient demographic and risk factor data from our telestroke log and our hub hospital electronic medical record. CTA utilization was determined by telestroke consult log documentation because most SHs used a separate electronic medical record, which we did not have access to; for this reason, we were unable to include reliable door-in and door-out times. Patients with CTA recommended but not documented were defined as not undergoing CTA imaging. An ELVO was confirmed if vessel imaging performed at the $\mathrm{SH}$ showed an occlusion in the intracranial ICA, MCA M1 segment, proximal M2 segment, or basilar artery with NIHSS score $\geq 6$ points and LKW $<24$ hours. Tandem lesions involving cervical ICA stenosis or occlusion with an intracranial occlusion were
Table 1: Patient demographics pre- and post-CTA protocol implementation $^{\mathrm{a}}$

\begin{tabular}{llcc}
\hline & $\begin{array}{c}\text { Pre-CTA, } \\
\boldsymbol{n}=167(\%)\end{array}$ & $\begin{array}{c}\text { Post-CTA, } \\
\boldsymbol{n}=\mathbf{2 0 7}(\%)\end{array}$ & $\begin{array}{c}\boldsymbol{P} \\
\text { Value }\end{array}$ \\
\hline Characteristics & & & \\
Age, median (IQR) & $76(63,85)$ & $71(57,82)$ & .066 \\
Female sex & $88(53)$ & $113(55)$ & .755 \\
White race & $151(90)$ & $88(84)$ & .127 \\
Risk factors & & & \\
Atrial fibrillation & $54(32)$ & $50(24)$ & .083 \\
Coronary artery disease & $42(25)$ & $28(14)$ & .005 \\
Diabetes mellitus & $26(16)$ & $46(22)$ & .115 \\
Dyslipidemia & $43(26)$ & $53(26)$ & 1.000 \\
Heart failure & $12(7)$ & $15(7)$ & 1.000 \\
Hypertension & $85(51)$ & $100(48)$ & .677 \\
Previous stroke or TIA & $41(25)$ & $59(29)$ & .413 \\
Smoking & $8(5)$ & $18(9)$ & .101 \\
\hline
\end{tabular}

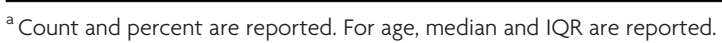

included. ${ }^{17}$ We defined late-window patients as $>4.5$ hours from LKW to consult start time at the $\mathrm{SH}$; this equated to roughly $>6$ hours at the time of arrival to an EVT capable center, allowing 1.5 hours for time for the evaluation and transport of the patient. For patients transferred to our hub, we analyzed rates of additional imaging after arrival, EVT, and utilization of a direct-to-EVT protocol, which allowed transferred patients with confirmed ELVO to bypass both the emergency department and further imaging to proceed directly to the endovascular suite for thrombectomy. The overall rate of large-vessel occlusion (LVO) transferred was defined as the total number of LVOs identified at the SH in addition to LVOs identified at the hub that were not previously identified at the $\mathrm{SH}$ among patients transferred to the hub hospital.

This study was approved by the local institutional review board. Median and interquartile range were reported for continuous variables. Percent and count were reported for categoric variables. Differences were assessed by using nonparametric Wilcoxon rank-sum for continuous variables and Fisher exact tests for categoric variables. Two-tailed $P$ values $<.05$ were interpreted as statistically significant. Analyses were performed with SPSS version 23.0 (IBM). The data that support the findings of this study are available from the corresponding author upon request.

\section{RESULTS}

There were 167 patients pre-CTA and 207 patients post-CTA from 22 SHs. These cohorts were broadly similar (Table 1). The median ages in the pre-CTA and post-CTA groups were 76 years and 71 years, respectively $(P=.066)$. Most patients were white $(88 \%)$. There were no differences in risk factors between the 2 groups, with the exception of coronary artery disease (25\% preCTA versus $14 \%$ post-CTA; $P=.005)$. The proportion of offhour consults was similar at approximately 30\%. The median NIHSS score was 12 points for both groups, and no significant differences in rates of tPA administration were present (Table 2). There were fewer than 5 posterior circulation LVOs in each cohort.

The rate of CTA utilization at $\mathrm{SHs}$ increased significantly from $25 / 167(15 \%)$ to $144 / 207(70 \%)(P<.001)$ (Table 2). Three 
patients in the pre-CTA group had MRA rather than CTA. Reasons for not obtaining a CTA in the post-CTA cohort included known allergy to iodinated contrast (5 patients), renal dysfunction (4 patients), technical difficulties (3 patients; 1 unclear reasons, 1 lack of radiology technicians overnight, and 1 inability to obtain intravenous access), high suspicion for an alternative diagnosis (13 patients, including seizure, toxic-metabolic encephalopathy, or functional neurologic disorder), and determination of EVT ineligibility based on other factors (1 patient). Technical difficulties preventing CTA acquisition represented only $1.4 \%$ of cases after protocol implementation. The rates of late-window ( $>4.5$ hours from LKW) consults also increased significantly from $14 / 167$ (8\%) to $42 / 207$ (20\%) $(P=.001)$. Despite increased screening of patients in this late window, the rates of patient transfer out of SHs remained stable (93/167, 56\% versus $112 / 207,54 \% ; P=.83)$. This rate of transfer encompasses patients with ELVO who were transferred to another thrombectomy-capable center, as well as patients transferred to our center for other reasons, including seeking a higher level of care, such as a neurologic intensive care unit, seeking care for a nonstroke diagnosis, or to complete a stroke work-up. The rate of transfer to our hub hospital specifically for EVT showed a nonsignificant increase $(46 / 167,26 \%$ versus $73 / 207,35 \%$; $P=.119$ ), but the proportion of patients transferred in the late window increased nearly 5 -fold $(3 / 46,7 \%$ versus $25 / 73,34 \%$; $P<.001)$. Of those transferred to our hub hospital as ELVO, the overall LVO identification rate, whether imaged at the $\mathrm{SH}$ or hub, increased (67\% to $93 \%$; $P=.001)$ (Table 3$)$. Furthermore,

Table 2: Clinical, imaging, and transfer variables pre- and postCTA protocol implementation $^{\mathrm{a}}$

\begin{tabular}{lccc}
\hline & $\begin{array}{c}\text { Pre-CTA, } \\
\boldsymbol{n = 1 6 7}(\%)\end{array}$ & $\begin{array}{c}\text { Post-CTA, } \\
\boldsymbol{n = 2 0 7}(\%)\end{array}$ & $\begin{array}{c}\boldsymbol{P} \\
\text { Value }\end{array}$ \\
\hline NIHSS score at SH (IQR) & $12(8-19)$ & $12(8-18)$ & .678 \\
Late window & $14(8)$ & $42(20)$ & .001 \\
Off hours (7 PM-7 AM) & $53(32)$ & $61(30)$ & .653 \\
CTA performed at SH & $25(15)^{\mathrm{b}}$ & $144(70)$ & $<.001$ \\
LVO identified at SH & $13(8)$ & $81(39)$ & $<.001$ \\
tPA administered at SH & $93(60)$ & $96(68)$ & .185 \\
Transferred to hub & $46(26)$ & $73(35)$ & .119 \\
$\quad$ hospital & & & \\
Retained at SH & $74(44)$ & $95(46)$ & .834 \\
Retained at SH, of those & $65(43)$ & $81(49)$ & .260 \\
$\quad$ in early window & & &
\end{tabular}

${ }^{a}$ Count and percent are reported.

${ }^{b}$ Includes 3 patients who underwent MRA. Late window was defined as $>4.5$ hours from LKW at telestroke consult start time.

Table 3: Processing of transferred patients at the hub hospital ${ }^{\mathrm{a}}$

\begin{tabular}{lccc}
\hline & $\begin{array}{c}\text { Pre-CTA, } \\
\boldsymbol{n}=\mathbf{4 6}(\%)\end{array}$ & $\begin{array}{c}\text { Post-CTA, } \\
\boldsymbol{n}=\mathbf{7 3}(\%)\end{array}$ & $\boldsymbol{P}$ Value \\
\hline Late window & $3(7)$ & $25(34)$ & $<.001$ \\
Overall LVO transferred to hub & $31(67)$ & $68(93)$ & .001 \\
Repeat imaging, overall & $43(94)$ & $48(66)$ & $<.001$ \\
Repeat imaging, early window & $39(91)$ & $24(50)$ & $<.001$ \\
Direct-to-EVT protocol utilization & 0 & $20(27)$ & $<.001$ \\
Received EVT, overall & $10(22)$ & $34(47)$ & .011 \\
Received EVT, early window & $9(21)$ & $25(52)$ & .004 \\
\hline
\end{tabular}

${ }^{a}$ Repeat hub imaging, direct-to-EVT protocol utilization, and EVT for patients transferred to our institution from an SH with NIHSS scores $\geq 6$ points for consideration of EVT. there was a decrease in the need for repeat imaging at our hub hospital (43/46, 94\% versus 48/73, 66\%; $P<.001$ ). For the 48 patients in the post-CTA cohort who required repeat imaging, $34 / 48$ (71\%) required imaging to confirm mismatch before proceeding with late-window thrombectomy. A total of 24/34 (71\%) were already considered late window by our criteria before transfer, and 10/34 (29\%) were considered early window. Repeat imaging was required in 3/48 (6\%) cases for worsening NIHSS score. The most common technique for repeat imaging was MR imaging with or without MRA in 42/48 (88\%). In the pre-CTA cohort, $14 / 46$ patients $(30 \%)$ without a CTA at the $\mathrm{SH}$ were transferred to our center for EVT evaluation but were not found have an LVO on their hub hospital CTA or MRA imaging. The overall rate of EVT performed on patients transferred to our hub for possible EVT more than doubled $(10 / 46,22 \%$ to $34 / 73,47 \%$; $P=.011)$. In the post-CTA cohort, 20/73 (27\%) of transferred patients were processed via our direct-to-EVT protocol (this protocol was introduced in December 2017 and therefore did not exist in the pre-CTA era).

Our post-CTA cohort spanned a timeframe after which the late-window thrombectomy trials were published and the potential pool of EVT candidates was increased. Given these differences, to better compare our 2 cohorts, we performed sensitivity analyses based on the early-window patients. The transfer rate was nonsignificantly decreased after implementation of the CTA protocol $(88 / 153,57 \%$ to $84 / 164,51 \%$; $P=.26)$. The decrease in repeat imaging after transfer was notably even greater for patients transferred in the early window (39/43, 91\% versus $24 / 48,50 \%$; $P<.001)$. The rate of EVT performed at our center for patients in the early window increased significantly $(9 / 43,21 \%$ to $25 / 48$, $52 \% ; P=.004)$.

\section{DISCUSSION}

These findings demonstrate the successful implementation of a systematic CTA protocol performed at SHs within our telestroke network and its impact on SH retention rates and hub hospital EVT rates. Implementation of a CTA protocol proved feasible. Provision to SHs of a standardized imaging protocol and the use of cloud-based image sharing proved useful in maintaining image quality across the network. The rate of CTA utilization at SHs increased from $15 \%$ to $70 \%$ during the study period, with multiple gains in system efficiency.

Most patients with acute ischemic strokes, including ELVOs, present to nonthrombectomy-capable hospitals. ${ }^{11}$ Telestroke networks therefore have a crucial role in not only delivering care, including thrombolytics, but also quickly and cost-effectively triaging patients who may need EVT. ${ }^{1}$ The ideal triage system is uncertain, and debate remains regarding different triage strategies, which likely depend on geographic region. ${ }^{18}$ A persistent challenge is the low rates of ELVO among general stroke presentations. ${ }^{19}$ Currently, randomized trials are evaluating the triage option of bypassing the $\mathrm{SH}$ for patients whom emergency first responders in the field suspect harbor an ELVO. ${ }^{20-22}$ The possibility of $\mathrm{SH}$ bypass, though promising, has raised 
concerns regarding care quality at both $\mathrm{SH}$ (decreased patient volumes and local expertise) and hub hospitals (excessive patient volumes straining resources). ${ }^{18,23}$ The expansion of the treatment window for thrombectomy to 24 hours raises further unique concerns with triage, given the expanded pool of patients to be evaluated for a progressively smaller number of treatment candidates. $^{24}$

One potential system solution is to refine triage at the level of the SH by improved identification of patients with ELVO. This refinement and reinforcement of existing care models seeks to improve overall system efficiency. A primary reason patients transferred to a hub hospital for EVT do not ultimately undergo thrombectomy is that no ELVO is present despite a suggestive clinical presentation. ${ }^{25-27}$ This was confirmed in our pre-CTA cohort in which one-third of patients transferred to our hub did not have an LVO. Identifying which patients do not require EVT while still at the $\mathrm{SH}$ represents, therefore, a potential intervention in the evolution of stroke care systems. Although several available imaging modalities exist, ELVO identification is most accurately accomplished by using CTA because of both rapid image acquisition and high sensitivity and specificity. ${ }^{28}$ Triage with CTA at the level of the $\mathrm{SH}$ allows stroke care to remain local for patients without ELVO while transporting confirmed patients with ELVO to appropriately enabled care environments. The impact of CTA for all patients with stroke is being increasingly defined at thrombectomy capable centers. A health system in Detroit expanded CTA imaging to all patients with stroke after previously reserving it for patients with NIHSS scores $\geq 6$ points. ${ }^{29}$ CTA scanning for all suspected patients with stroke led to increased rates of ELVO detection, increased mechanical thrombectomy treatment, and a possible trend toward improved outcomes in that system (a single comprehensive stroke center hub and 8 associated hospitals). By comparison, this present study is novel in describing the effects of CTA triage in SHs within an entire, widely distributed telestroke network where CTA imaging had not been routinely performed previously.

The long-term effects of late-window EVT trials on telestroke networks remain unknown. Our findings indicate an expansion of the pool of candidates evaluated, driven predominantly by the large increase in late-window candidates. However, despite the additional patients screened in the post-CTA cohort, the overall rate of transfers from SHs was similar (56\% versus 54\%). This is noteworthy given the anticipated higher transfer numbers based on clinical grounds alone that are expected with the expansion of the thrombectomy window to 24 hours. In the pre-CTA era, patients $>6$ hours from onset would have been deemed nonthrombectomy candidates. With the expanded window, these patients would be expected to transfer for evaluation for thrombectomy if clinical criteria alone were used. The use of CTA at SHs allowed screening at the source to select appropriate candidates from this larger pool. CTA application demonstrated a trend toward decreasing transfers in the early window after the protocol was implemented (57\% versus 51\%), but concurrently, rates of patients transferred to our center $>4.5$ hours from LKW increased nearly 5-fold as extended-window evaluation was increasingly implemented across the $\mathrm{SH}$ network. The decrease in futile transfers therefore helped balance the expected rise in transfers to keep the retention rate stable in the pre- and postCTA periods. We anticipate the proportion of late-window transfers to continue to increase with increasing awareness regarding late-window therapy for patients with ELVO and with revisions to existing $\mathrm{SH}$ emergency protocols.

Patients with ELVO require specialized attention, which in addition to EVT may include support in a neurologic critical care unit or open surgical interventions (hemicraniectomy or suboccipital craniectomy). Comprehensive stroke centers, such as hub hospitals in telestroke networks, are best equipped to manage these patients. ${ }^{30,31}$ The rates of EVT performed on transferred patients more than doubled from $22 \%$ pre-CTA to $47 \%$ in the post-CTA cohort. This finding was also observed when analyzing early-window patients alone ( $21 \%$ versus $52 \%$ ), supporting the fact that this was not driven by the increase in late-window patients. Reduction in false activation of the EVT treatment team represents appreciable resource conservation for the hub hospital. In addition, the need for repeat imaging was decreased as ELVO status was established before hub hospital arrival, allowing the use of direct-to-EVT protocols that have been proved to save time in other practice settings. ${ }^{32,33}$ This was true for $27 \%$ of our transferred population undergoing thrombectomy. For patients in the early window, CTA at the $\mathrm{SH}$ represents a task shifted from the hub hospital, often obviating the need for imaging at the hub if transfer occurs expeditiously. Accelerating transfer between $\mathrm{SH}$ and hub ensures that imaging performed at the $\mathrm{SH}$ is temporally relevant to hub hospital treatment decisions. For latewindow patients, although CTA screens for the presence of ELVO, it does not provide the additional information necessary for guideline-based treatment decisions (core volume, perfusion deficit, mismatch volume, and so on). ${ }^{16,34}$ These patients therefore require additional imaging on arrival at the hub.

The ability to shift imaging tasks from the hub to the $\mathrm{SH}$ has additional implications for future efforts in ELVO triage and EVT selection. Automation of stroke imaging, already established for CT perfusion, shows nascent promise for ELVO detection and other components of stroke imaging. ${ }^{35-37}$ The role of this software in the SH environment may expand with time and remains to be explored as the software becomes more available, affordable, and refined. The implications for automation and coordination of triage in a stroke system of care may be large, with telestroke networks well poised to pioneer these approaches.

There are several limitations to our study, mostly related to its retrospective design. The late-window thrombectomy trials, which resulted in more patients being eligible for EVT and in an increasing awareness of ELVOs, were published before our postCTA cohort. However, our sensitivity analyses included only patients in the early window, thereby making the cohorts more comparable, and did not change interpretation of the results. We relied on data that were available in clinical documentation. Furthermore, we selected an NIHSS score threshold of 6 points at the SH based on our clinical practice and guideline recommendations but recognize some patients with ELVOs may have had NIHSS scores $<6$ points and may not be captured in these data. ${ }^{29}$ A limited number of patients with high NIHSS scores or ELVOs were transferred to other thrombectomy centers, and we do not 
have details about what proportion of these patients later had confirmed ELVOs or underwent EVT. We focus on system processes and therefore do not detail patient outcomes because of limitations in data available in both the pre-CTA cohort and for patients with confirmed ELVOs transferred to other centers.

Finally, although we successfully implemented a CTA protocol at SHs, we are unable to compare time metrics at SHs, such as door-in-door-out and transport times, because SHs used a separate electronic medical record, and documentation of these metrics was not a requisite in our telestroke log or consistent in available documentation. Acquisition of the CTA immediately after the head CT without returning the patient to the emergency department is an important part of the CTA imaging protocol to ensure time savings, but implications for tPA delivery times remain to be explored. However, we present a practical and real-world experience that details the response to $\mathrm{SH}$ imaging triage in a telestroke network.

\section{CONCLUSIONS}

CTA application in a standardized fashion to the imaging protocols of SHs in a large telestroke network was feasible. Adoption was high within 1 year of protocol implementation and added important additional screening information for potential patients with ELVO at SHs. A standardized CTA protocol at SHs was associated with increased telestroke network efficiency by increasing the proportion of transferred patients who undergo EVT, thereby decreasing futile transfers. The retention rate for $\mathrm{SHs}$ remained relatively stable despite higher numbers of patients screened (partly caused by a 5-fold expansion of late-window candidates). For $27 \%$ of transferred patients undergoing thrombectomy, this shift in imaging confirmation to the SH enabled a direct-to-EVT protocol, eschewing emergency department evaluation and further imaging at the hub hospital. Future evaluation for such system evolutions will include analysis of transfer times and an economic analysis of the economic consequences. Continued research to identify optimized triage in stroke care systems is needed.

Disclosures: Robert Regenhardt-RELATED: Grant: NIH NINDS, Comments: R25 NS065743*. Lee Schwamm—RELATED: Grant: NINDS; UNRELATED: Consultancy: Genentech, Lifelmage, Mass DPH, Penumbra, Diffusion Pharma, Comments: scientific consultant regarding trial design and conduct to Genentech (late-window thrombolysis) and member of steering committee (TIMELESS NCT03785678), consultant on user interface design and usability to Lifelmage, stroke systems of care consultant to the Massachusetts Department of Public Health, and member of a Data Safety Monitoring Boards (DSMB) for Penumbra (MIND NCT03342664) and for Diffusion Pharma PHAST-TSC NCT03763929); Grants/Grants Pending: NINDS, Genentech, Medtronic, Comments: National Co-PI, late-window thrombolysis trial, NINDS (P50NS051343, MR WITNESS NCT01282242, and alteplase provided free of charge to Massachusetts General Hospital as well as supplemental per-patient payments to participating sites by Genentech), Site Pl, StrokeNet Network NINDS (New England Regional Coordinating Center U24NS107243), National PI for Medtronic (Stroke AF NCT02700945)*. Aman Patel—UNRELATED: Consultancy: Penumbra, Microvention, Medtronic; Grants/Grants Pending: Siemens. Anand Viswanathan-UNRELATED: Consultancy: Alynylam Pharmaceuticals, Biogen Pharmaceuticals, Roche; Grants/Grants Pending: NIH*. Joshua Hirsch—UNRELATED: Consultancy: Medtronic, Relevant Medsystems; Grants/Gants Pending: Neiman Health Policy Institute. Michael Lev-UNRELATED: Consultancy: Takeda, GE Healthcare; Grants/Grants Pending: GE, NIH-institutional. *Money paid to institution.

\section{REFERENCES}

1. Akbik F, Hirsch JA, Chandra RV, et al. Telestroke-the promise and the challenge. Part one: growth and current practice. J Neurointerv Surg 2017;9:357-60 CrossRef Medline

2. Akbik F, Hirsch JA, Chandra RV, et al. Telestroke-the promise and the challenge. Part two-expansion and horizons. J Neurointerv Surg 2017;9:361-65 CrossRef Medline

3. Leslie-Mazwi T, Chandra RV, Baxter BW, et al. ELVO: an operational definition. J Neurointerv Surg 2018;10:507-09 CrossRef Medline

4. Malhotra K, Gornbein J, Saver JL. Ischemic strokes due to large-vessel occlusions contribute disproportionately to stroke-related dependence and death: a review. Front Neurol 2017;8:651 CrossRef Medline

5. Regenhardt RW, Takase H, Lo EH, et al. Translating concepts of neural repair after stroke: structural and functional targets for recovery. Restor Neurol Neurosci 2020;38:67-92 CrossRef Medline

6. Regenhardt RW, Biseko MR, Shayo AF, et al. Opportunities for intervention: stroke treatments, disability and mortality in urban Tanzania. Int J Qual Health Care 2019;31:385-92 CrossRef Medline

7. Sheth SA, Jahan R, Gralla J, et al. Time to endovascular reperfusion and degree of disability in acute stroke. Ann Neurol 2015;78:584-93 CrossRef Medline

8. Jahan R, Saver JL, Schwamm LH, et al. Association between time to treatment with endovascular reperfusion therapy and outcomes in patients with acute ischemic stroke treated in clinical practice. JAMA 2019;322:252-63 CrossRef Medline

9. Regenhardt RW, Das AS, Stapleton CJ, et al. Blood pressure and penumbral sustenance in stroke from large vessel occlusion. Front Neurol 2017;8:317 CrossRef Medline

10. Seners P, Turc G, Naggara O, et al. Post-thrombolysis recanalization in stroke referrals for thrombectomy: incidence, predictors, and prediction scores. Stroke 2018;49:2975-82 CrossRef Medline

11. Katz BS, Adeoye O, Sucharew H, et al. Estimated impact of emergency medical service triage of stroke patients on comprehensive stroke centers: an urban population-based study. Stroke 2017;48:2164-70 CrossRef Medline

12. Goyal M, Menon BK, van Zwam WH, et al. Endovascular thrombectomy after large-vessel ischaemic stroke: a meta-analysis of individual patient data from five randomised trials. Lancet 2016;387:172331 CrossRef Medline

13. Albers GW, Marks MP, Kemp S, et al. Thrombectomy for stroke at 6 to 16 hours with selection by perfusion imaging. $N$ Engl J Med 2018;378:708-18 CrossRef Medline

14. Nogueira RG, Jadhav AP, Haussen DC, et al. Thrombectomy 6 to 24 hours after stroke with a mismatch between deficit and infarct. $N$ Engl J Med 2018;378:11-21 CrossRef Medline

15. Jauch EC, Saver JL, Adams HP Jr, et al. Guidelines for the early management of patients with acute ischemic stroke: a guideline for healthcare professionals from the American Heart Association/ American Stroke Association. Stroke 2013;44:870-947 CrossRef Medline

16. Powers WJ, Rabinstein AA, Ackerson T, et al. Guidelines for the Early Management of Patients With Acute Ischemic Stroke: 2019 Update to the 2018 Guidelines for the Early Management of Acute Ischemic Stroke: A Guideline for Healthcare Professionals From the American Heart Association/American Stroke Association. Stroke 2019;50:e344-418 CrossRef Medline

17. Nolan NM, Regenhardt RW, Koch MJ, et al. Treatment Approaches and Outcomes for Acute Anterior Circulation Stroke Patients with Tendem Lesions. J Stroke Cerebrovasc Dis 2021;30:105478 CrossRef Medline

18. Southerland AM, Johnston KC, Molina CA, et al. Suspected large vessel occlusion: should emergency medical services transport to the nearest primary stroke center or bypass to a comprehensive stroke center with endovascular capabilities? Stroke 2016;47:196567 CrossRef Medline 
19. Raychev RI, Stradling D, Patel N, et al. Evolution of a US county system for acute comprehensive stroke care. Stroke 2018;49:1217-22 CrossRef Medline

20. Froehler MT, Saver JL, Zaidat OO, et al. Interhospital transfer before thrombectomy is associated with delayed treatment and worse outcome in the STRATIS Registry (Systematic Evaluation of Patients Treated With Neurothrombectomy Devices for Acute Ischemic Stroke). Circulation 2017;136:2311-21 CrossRef Medline

21. Abilleira S, Perez de la Ossa N, Jimenez X, et al. Transfer to the Local Stroke Center versus Direct Transfer to Endovascular Center of Acute Stroke Patients with Suspected Large Vessel Occlusion in the Catalan Territory (RACECAT): study protocol of a cluster randomized within a cohort trial. Int J Stroke 2019;14:734-44 CrossRef Medline

22. Behrndtz A, Johnsen SP, Valentin JB, et al. TRIAGE-STROKE: Treatment strategy In Acute larGE vessel occlusion: prioritize IV or endovascular treatment-a randomized trial. Int J Stroke 2020;15:103-08 CrossRef Medline

23. Reznek MA, Murray E, Youngren MN, et al. Door-to-imaging time for acute stroke patients is adversely affected by emergency department crowding. Stroke 2017;48:49-54 CrossRef Medline

24. Adeoye O, Nystrom KV, Yavagal DR, et al. Recommendations for the establishment of stroke systems of care: a 2019 update. Stroke 2019;50:e187-210 CrossRef Medline

25. Leslie-Mazwi TM, Hirsch JA, Falcone GJ, et al. Endovascular stroke treatment outcomes after patient selection based on magnetic resonance imaging and clinical criteria. JAMA Neurol 2016;73:43-49 CrossRef Medline

26. Yi J, Zielinski D, Ouyang B, et al. Predictors of false-positive stroke thrombectomy transfers. J NeuroIntervent Surg 2017;9:834-36 CrossRef Medline

27. Regenhardt RW, Mecca AP, Flavin SA, et al. Delays in the air or ground transfer of patients for endovascular thrombectomy. Stroke 2018;49:1419-25 CrossRef Medline
28. Verro P, Tanenbaum LN, Borden NM, et al. CT angiography in acute ischemic stroke: preliminary results. Stroke 2002;33:276-78 CrossRef Medline

29. Mayer SA, Viarasilpa T, Panyavachiraporn N, et al. CTA-for-all: impact of emergency computed tomographic angiography for all patients with stroke presenting within 24 hours of onset. Stroke 2020;51:331-34 CrossRef Medline

30. Ali SF, Singhal AB, Viswanathan A, et al. Characteristics and outcomes among patients transferred to a regional comprehensive stroke center for tertiary care. Stroke 2013;44:3148-53 CrossRef Medline

31. Meretoja A, Roine RO, Kaste M, et al. Effectiveness of primary and comprehensive stroke centers: PERFECT stroke: a nationwide observational study from Finland. Stroke 2010;41:1102-07 CrossRef Medline

32. Jadhav AP, Kenmuir CL, Aghaebrahim A, et al. Interfacility transfer directly to the neuroangiography suite in acute ischemic stroke patients undergoing thrombectomy. Stroke 2017;48:1884-89 CrossRef Medline

33. Mendez B, Requena M, Aires A, et al. Direct transfer to angio-suite to reduce workflow times and increase favorable clinical outcome. Stroke 2018;49:2723-27 CrossRef Medline

34. Saposnik G, Menon BK, Kashani N, et al. Factors associated with the decision-making on endovascular thrombectomy for the management of acute ischemic stroke. Stroke 2019;50:2441-47 CrossRef Medline

35. Vagal A, Wintermark M, Nael K, et al. Automated CT perfusion imaging for acute ischemic stroke: pearls and pitfalls for realworld use. Neurology 2019;93:888-98 CrossRef Medline

36. Amukotuwa SA, Straka M, Dehkharghani S, et al. Fast automatic detection of large vessel occlusions on CT angiography. Stroke 2019;50:3431-38 CrossRef Medline

37. Feng R, Badgeley M, Mocco J, et al. Deep learning guided stroke management: a review of clinical applications. J Neurointerv Surg 2018;10:358-62 CrossRef Medline 